\title{
LA LÓGICA DE IMPLICACIÓN NORMATIVA DE VON WRIGHT Y LA PARADOJA DE CHISHOLM*
}

\author{
Juliano Maranhão \\ Universidad de São Paulo
}

RESUMEN. En sus últimos trabajos sobre lógica deóntica VON WRIGHT reinterpreta sus teoremas y derivaciones como meros patrones de racionalidad legislativa, sin compromiso con la existencia de las normas derivadas. Esta propuesta utiliza una cuasi-semántica, sin recurso a valores de verdad, que siguiendo a HANSEN (1999) llamaremos lógica de implicación normativa (LIN). En LIN VON WRIGHT rescata al sistema estándar y sugiere que las normas condicionales podrían ser representadas por una implicación material dentro del alcance del operador deóntico sin caer en conocidas paradojas, en especial la paradoja de CHISHOLM (obligaciones contrarias al deber). En este artículo confronto LIN con la paradoja de CHISHOLM, identifico algunas limitaciones frente a los requisitos de adecuación de CARMO y JONES (2002) y apunto dos dificultades (el problema de la inconsistencia racional y del Kindergarten) que desafían el uso de la implicación material. Seguido a ello, ofrezco una reconstrucción diádica de LIN que supera esas limitaciones y dificultades.

Palabras claves: lógica deóntica, condicionales, vON WRIGHT, paradoja de CHISHOLM, obligaciones contrarias al deber.

\section{Von Wright's Logic of Normative Implication and Chisholm Paradox}

ABSTRACT. VON WRIGHT's last approach to deontic logic reinterprets its theorems and derived norms as mere standards of rational legislation, without any ontological commitment. This proposalwhich, following HANSEN we shall call «logic of normative implication» (LIN) employs a semantics with no appeal to truth-values. Within LIN vON WRIGHT suggests that conditional norms could be represented by a material implication in the scope of the deontic operators, without raising well known paradoxes such as CHISHOLM's (contrary to duty obligations). The paper confronts LIN with CHISHOLM's paradox and identifies some limitations considering CARMO and JONES (2002) adequacy conditions (the problem of rational inconsistency and the one called the «Kindergarten problem»), which challenge the use of material implication. The paper then offers a dyadic reconstruction of LIN overcoming these limitations.

Keywords: deontic logic, VON WRIGHT, conditionals, CHISHOLm's paradox, contrary to duty obligations.

\footnotetext{
* Fecha de recepción: 29 de junio de 2016. Fecha de aceptación: 1 de septiembre de 2016.
} 


\section{EL ESCEPTICISMO DE VON WRIGHT Y SU LÓGICA DE IMPLICACIÓN NORMATIVA}

V

ON WRIGHT es considerado como el padre de la lógica deóntica. No solo resucitó el tema sino que también en las décadas posteriores hizo importantes contribuciones técnicas para su desarrollo, e.g., una lógica de la acción como fragmento de la lógica deóntica y una propuesta de sistemas deónticos diádicos. Pero, pocas veces se vio a un padre tan motivador y al mismo tiempo tan angustiado con su criatura. VON WRIGHT siempre estuvo preocupado por una fundamentación filosófica de las lógicas normativas y vivió perturbado con la idea de que esas lógicas ni siquiera serían posibles como representaciones adecuadas del discurso normativo.

Acompañar esta peregrinación no es solo una fuente de aprendizaje de la lógica deóntica y de los problemas filosóficos en torno de ella sino también una lección sobre cómo hacer filosofía. VON WRIGHT acostumbraba a decir que estudiaba los textos filosóficos para encontrar rompecabezas y problemas, y entonces pensar sobre ellos de manera independiente. Lo mismo valía para sus propios textos. Sus artículos reflejan exactamente ese modo de hacer filosofía, siempre con una introducción clara sobre los problemas que se enfrentan, invitando al lector a pensarlos libremente.

Fue con ese espíritu que conocí al profesor VON WRIGHT. Al final de mi graduación en derecho le escribí para exponerle una duda sobra la adecuación de su reinterpretación de la lógica deóntica para dar cuenta de los problemas de las lagunas en los sistemas normativos. Su reacción no fue la de corregir o resolver mi duda, sino de reconocer una cuestión nueva y digna de reflexión, lo que complementó con nuevos desafíos. Una respuesta a esos desafíos resultó en la publicación de un artículo (MARANHÃO, 2002).

Poco tiempo después hice las maletas y viajé a Finlandia, donde pasé meses mágicos con discusiones sobre su último abordaje a la lógica deóntica (VON WRIGHT, $1983 ; 1985 ; 1991 ; 1996 ; 1997 ; 1999 a ; 1999 b)$. Esa perspectiva es consistente con la tesis de que no hay una genuina lógica de normas. Mi pregunta principal no era acerca de los sistemas de lógica de normas allí discutidos sino en el aparente cambio de método, o en la propia concepción de la lógica y cuál es su papel frente al discurso normativo.

Esas discusiones en la nevada Helsinki, instigadas una y otra vez por preguntas claves de VON WRIGHT, que incluyeron una invitación a estudiar WITTGENSTEIN, me acompañaron por algunos años y resultaron en un nuevo trabajo (MARANHÃO, 2009) donde defendí que ese escepticismo no es propiamente un decreto contra una lógica de normas sino un esfuerzo «terapéutico», en el sentido del segundo WITTGENSTEIN, provocado sobre todo por el dilema de JORGENSEN (JORGENSEN, 1937-1938).

Al final de una larga peregrinación, VON WRIGHT propone que en lugar de interpretar los teoremas deónticos como un reflejo de las relaciones necesarias entre normas, o de la estructura del discurso prescriptivo, deberíamos tomarlo como meros patrones de racionalidad, patrones de lo que sería una actividad de legislación racional. Esa reinterpretación consiste básicamente en redefinir la relación de consecuencia nor- 
mativa (entre conjuntos de normas y una norma), sin recurso a valores de verdad, por medio de las nociones de consistencia y de norma-negación, en correspondencia con el discurso prescriptivo ordinario. Una norma es consecuencia de (normativamente implicada por) un conjunto de normas si su norma-negación —o sea, el opuesto de lo que fue exigido por ella - fuese normativamente inconsistente con ese conjunto. Eso no significa que la norma derivada sea necesariamente válida o existente, tal como el conjunto de normas originales. Solo quiere decir que sería irracional legislar de otra manera (lo que no impide que el legislador concreto efectivamente lo sea). Al aligerar de ese modo la carga ontológica de los teoremas de la lógica deóntica y de la relación de consecuencia normativa, VON WRIGHT pretendía curar a los lógicos deónticos de sus angustias frente al carácter no-teórico de las normas.

Sin embargo, aunque VON WRIGHT había conseguido dar sentido a esas relaciones de contradicción y consecuencia entre normas, ciertas dificultades para interpretar conectivas proposicionales entre formulaciones de normas que, en su opinión, solo tendrían sentido en una interpretación descriptiva, le llevó a excluir tales construcciones del conjunto de fórmulas bien formadas. Para VON WRIGHT, los sistemas que pretendiesen representar una lógica de normas genuinas (en términos de patrones de racionalidad) deberían satisfacer tal restricción (VON WRIGHT, 1991; 1996).

Así, las definiciones de consistencia y de implicación normativa ofrecen una relación semántica de consecuencia solamente entre formulaciones normativas. Junto con esa restricción, una vieja tesis, ya presente en su sistema original (VON WRIGHT, 1951), fue rescatada: las obligaciones condicionales deberían ser representadas por una implicación material en el alcance de un operador deóntico.

Esa representación de la condicionalidad normativa es importante dentro de su perspectiva escéptica. Ello se debe a que la única relación de implicación con significado es aquella de implicación normativa (entre normas); por consiguiente, la implicación material no puede estar fuera del alcance de un operador deóntico. Como justificación para ella, VON WRIGHT esgrime el argumento de que los problemas lógicos ligados a las formulaciones normativas condicionales pueden ser superados sin recurrir a una teoría diádica.

Aunque las paradojas de ROSS y PRIOR se disuelven fácilmente (vid. MARANHÃO, 2009), la paradoja de Chisholm presenta más dificultades (HANSEN, 2001). Su solución, dentro de los esquemas de la lógica de implicación normativa, involucra algunos recursos adicionales. Pero, aparentemente, hay una falta de adecuación entre la definición de consistencia de VON WRIGHT y la representación de los conflictos de normas condicionales.

La tentativa de adaptar su test de consistencia normativa para captar conflictos de obligaciones condicionales necesariamente desnaturaliza la implicación material, lo que mina el rescate puro y simple del sistema estándar (o de su fragmento estrictamente normativo). Aquí propondremos una adaptación del concepto de consistencia que genera una nueva lógica de implicación normativa diádica capaz de superar las inadecuaciones señaladas. 


\section{FORMALIZANDO LA LÓGICA DE IMPLICACIÓN NORMATIVA DE VON WRIGHT}

Para enfrentar el problema con el detalle y la profundidad necesarios, recurriremos a una formulación más precisa de la lógica de implicación normativa (LIN) propuesta por VON WRIGHT.

El lenguaje $L$ de LIN contiene un conjunto numerable de letras proposicionales $p$, $q, r . .$, las conectivas proposicionales $« \sim, \wedge, \vee, \rightarrow »$, respectivamente, para la negación, conjunción, implicación material (la equivalencia, «↔», es definida de forma usual y $\ll \perp »$ es una constante de falsedad y una «taut $\gg$ es una constante de verdad) y los operadores «O» e «P», respectivamente, para obligación y permisión. Los conceptos básicos de la teoría de conjuntos, tales como la pertenencia, subconjunto, unión $(\in, \subseteq, \cup)$ serán utilizados en un nivel metalingüístico. El símbolo « $\Rightarrow »$ representa la relación de consecuencia clásica, también en el metalenguaje (a lo largo de la discusión definiremos otras relaciones de consecuencia). El conjunto de fórmulas es construido en dos pasos. Primero, el conjunto $F_{C}$ de contenidos normativos se forma con el conjunto de todas las fórmulas proposicionales clásicas. El conjunto $F_{N}$ de formulaciones normativas se forma a partir de las siguientes cláusulas:

(i) $\mathrm{Si} a$ es un contenido normativo (i. e. $a \in F_{C}$ ) entonces $\mathrm{O} a$ e $\mathrm{Pa}$ son formulaciones normativas (i.e. $\mathrm{O} a, \mathrm{P} a \in F_{N}$ ).

(ii) $F_{N}$ es el menor conjunto que satisface (i) ${ }^{1}$.

De un conjunto $N$ de formulaciones normativas podemos obtener el conjunto de contenidos normativos correspondiente, definido como $N_{C}=\{a: O a \in N$ o $P a \in N\}$.

De acuerdo con la semántica descrita por VON WRIGHT, una condición mínima necesaria para la racionalidad del legislador es que sus normas sean ejecutables, es decir, todos los estados de cosas obligatorios deben poder ser realizados sin interferir con el uso de cualquier permiso. Esto nos lleva a la siguiente definición formal de consistencia deóntica.

Definición de consistencia deóntica. Sea $N$ un conjunto de formulaciones normativas. Entonces, $N$ es $d$-inconsistente (en fórmulas: $i n c d(N)$ ) si y solamente si, existe un conjunto $M=\{O a 1, O a 2, \ldots, O a n\} \subseteq N$ o $M=\{O a 1, O a 2, \ldots, O a n, P x\} \subseteq N$ tal que $M C \Rightarrow \perp$. En caso contrario, $N$ es $d$-consistente.

Los conjuntos consistentes, en el sentido antes definido, pueden ser llamados también «ejecutables». Nótese que la noción de consistencia, en el sentido de ser ejecutables, es distinta de las simples nociones de consistencia entre contenidos normativos. El conjunto $R=\{$ Obligatorio no fumar $(O \sim a)$, Permitido abrir la ventana $(P \sim b)$, Permitido no abrir la ventana $(\mathrm{Pb})\}$, que expresa la facultad de abrir la ventana junto a

\footnotetext{
${ }^{1}$ Por la definición de $F_{N}$ quedan excluidos del conjunto de formulaciones de normas cualquier fórmula compleja que combina fórmulas modales por medio de conectivas proposicionales, al igual que la reiteración de operadores modales, i. e. las fórmulas $O O a, O P b, P O a$, etc. No son fórmulas bien formadas.
} 
la prohibición de fumar es consistente según la definición, pero la conjunción de sus contenidos es inconsistente.

El test de consistencia da un tratamiento distinto entre obligaciones y permisiones al de la semántica de KRIPKE para el sistema estándar de lógica deóntica. Se verifica, simplemente, si cada una de las permisiones independientemente no entra en conflicto con el conjunto total de las obligaciones asumidas (así como el contenido de una permisión está únicamente en un mundo ideal accesible, el contenido de una obligación está en todos los mundos ideales).

El concepto de racionalidad del legislador puede ser modificado para incluir restricciones más fuertes, tal como la exigencia de que los contenidos normativos sean no solamente consistentes (ejecutables) sino también que describan estados de cosas cuya realización sea física o humanamente posible. Si el conjunto de normas satisface esa exigencia adicional, llamaremos realizable al conjunto de normas. Todo conjunto de normas realizable es también ejecutable, pero obviamente la contraria no vale. Si la realizabilidad (y no la simple ejecutabilidad) fuese el patrón asumido de racionalidad, entonces las normas que exigen estados de cosas lógicamente necesarios o normas condicionales cuyas condiciones de aplicación fuesen física o humanamente imposibles serían marcas de la irracionalidad del legislador, siendo llamadas normas «espurias».

La norma «opuesta» a una cierta norma es introducida por VON WRIGHT mediante el concepto de norma-negación, que podemos formalizar como una función neg en el conjunto de formulaciones normativas $F_{N}$, tal que:

(i) $n e g(O a)=P \sim a$

(ii) $n e g(\mathrm{~Pa})=\mathrm{O} \sim a$

La implicación normativa es, entonces, obtenida a partir de las definiciones de norma-negación y consistencia deóntica.

Definición de implicación deóntica $\left(\Rightarrow_{d}\right)$. Sea $N$ un conjunto de formulaciones normativas y a una formulación normativa. Entonces, $N \Rightarrow_{d} \alpha$, si y sólo si, incd $(N \cup\{n e g(\alpha)\})$.

La formulación de la norma $\alpha$ es una tautología normativa si $\varnothing \Rightarrow_{d} \alpha$. Para simplificar la notación de un conjunto de premisas normativas unitario, i. e. que incluya solo una formulación normativa, omitiremos los paréntesis y escribiremos, por ejemplo, $\alpha \Rightarrow_{d} \beta$, donde $\alpha$ y $\beta$ son formulaciones normativas (en adelante, las letras latinas minúsculas denotan contenidos normativos y las letras griegas mayúsculas formulaciones normativas).

\section{FRENTE A LA PARADOJA DE CHISHOLM}

El esquema generador de la paradoja de Alf Ross en SDL, $O a \rightarrow O(a \vee b)$, corresponde a la relación de consecuencia $O a \Rightarrow d O(a \vee b)$, que es válida en LIN. La relación 
solo significa que $\{O a\} \cup\{P(\sim a \wedge \sim b)\}$ es $d$-inconsistente, i. e. si algo es obligatorio, entonces sería irracional permitir lo contrario conjuntamente con alguna otra cosa. Los teoremas de SDL que generan las paradojas de PRIOR son apenas variantes de la de Ross y, por ello, se disuelven con facilidad en LIN.

Sin embargo, LIN fue pensada por VON WRIGHT también con el objetivo de superar la paradoja de CHISHOLM ${ }^{2}$. Pero, su disolución dentro de LIN depende de algunas estrategias adicionales introducidas por VON WRIGHT. Veamos la paradoja.

\subsection{La paradoja}

Chisholm (Chisholm, 1963) llamó la atención sobre las dificultades para formalizar las obligaciones que surgen bajo condiciones contrarias al deber, es decir condiciones que son por sí mismas violaciones de otro deber. Esas normas son bastantes comunes en los ordenamientos jurídicos, dado que el legislador ha de tener en mente que los sujetos pueden desobedecer (y probablemente lo harán) algunos de sus deberes. Por ejemplo, para las leyes civiles brasileras está prohibido causar daños a terceros, pero si el daño ocurriese, entonces el responsable está obligado a resarcirlo. Por ello, la capacidad de lidiar con obligaciones contrarias al deber es importante para cualquier sistema de lógica deóntica.

Considérese el siguiente conjunto de proposiciones:

i) Es prohibido para John embarazar a Suzy Mae.

ii) Es obligatorio que si John no embaraza a Suzy Mae entonces no se case con ella.

iii) Si John embaraza a Suzy Mae, entonces es obligatorio que se case con ella.

iv) John embarazó a Suzy Mae.

Intuitivamente, esperamos derivar de esas premisas la obligación de John de casarse con Suzy Mae. También diríamos que esas premisas son lógicamente independientes. En SDL hay diferentes alternativas de formalización. Un primer intento considera las obligaciones expresadas en ii) y iii) como de tipos diferentes, dado que la última es una obligación con condición contraria a deber. Para captar esa diferencia y permitir la separación fáctica con la violación descrita en iv), se formulan las premisas en SDL de la siguiente manera:

1. $O \sim p$.

2. $O(\sim p \rightarrow \sim q)$.

3. $p \rightarrow O q$.

4. $p$.

Por modus ponens de 3 y 4 se deriva $O q$. El problema es que de 1 y 2 se deriva también $O \sim q$ en SDL, o sea una contradicción normativa, lo que no ocurre en el discurso ordinario. Se podría, entonces, criticar la distinción en la formalización de la obligación «regular» y de la obligación en condiciones «contrarias al deber».

${ }^{2}$ La solución para la paradoja de CHISHOLM dentro de los términos de la lógica de la implicación normativa es presentada de modo informal por VON WRIGHT en (1983: 157-159). 
La paradoja muestra la dificultad (vid. la discusión en LOwER y BELZER, 1983) en admitir en una misma lógica de obligación, la separación fáctica $[a, O(a \rightarrow b) \Rightarrow O b]$ y la separación deóntica $[\mathrm{O} a, \mathrm{O}(a \rightarrow b) \Rightarrow O b]$. LIN solo admite la separación deóntica.

Puede bien argumentarse que la diferencia gramatical en la formulación del ejemplo entre ii) y iii) es superficial y se debe seguir la propuesta de formalización de normas condicionales sustituyéndose 3 por

3. $O(p \rightarrow q)$

Esta formulación, en SDL, torna redundante la regulación 1-4, ya que 3' se sigue de 1 .

Por otro lado, si seguimos la sugerencia de PRIOR y formalizamos la obligación condicional como $p \rightarrow O q$, sustituyendo 2 por

2. $\sim p \rightarrow O \sim q$,

encontramos nuevamente redundancia en la formulación de las premisas ya que 2' se sigue de 4 .

Esa es la paradoja.

\subsection{La solución de VON WRIGHT}

VON WRIGHT creía que podría dar cuenta de la paradoja mediante sus definiciones intuitivas, que usamos para construir LIN. Solo tendríamos que formalizar las premisas i)-iv) usando 1, 2, 3' y 4 . El argumento de la redundancia sería inofensivo, ya que el hecho de que la norma 1 implique a 3', i. e. $O \sim p \Rightarrow{ }_{d} O(p \rightarrow q)$, no significa que «exista» la norma expresada por la fórmula, o sea que de la prohibición de embarazar a Suzy Mae se siga lógicamente la existencia de la obligación de John de casarse con ella si la embarazase, sino solo que prohibir a John embarazar a Suzy Mae - y al mismo tiempo- permitir embarazarla y no casarse con ella sería irracional, i. e., $\operatorname{inc}_{d}(\{O \sim p\} \cup\{P(p \wedge \sim q)\})$.

La única dificultad restante es la esperada separación fáctica a partir de la premisa 4. Es decir, aun cuando a partir de iii) y iv) se infiera que John debe casarse con Suzy Mae $O q$ no se sigue de 3' y 4 en LIN.

VON WRIGHT argumenta que el problema de la separación fáctico en una lógica normativa debe ser entendido desde el punto de vista del sujeto normativo, cuyo lenguaje de necesidades prácticas es distinta del discurso prescriptivo.

Una necesidad práctica o deber técnico («technical ought», representado $O_{t}$ ) es aquello que se requiere para la satisfacción de un determinado fin, es decir, es algo que precisa (tiene que) ser (hecho) para que se alcance un determinado objetivo. El deber deóntico ( «deontic ought», representado por $O_{d}$ ) es una prescripción de la autoridad normativa dirigida al sujeto normativo, i. e., aquello que es exigido por alguien y que, por tanto, debe ser (hecho). Las necesidades prácticas, al contrario de las prescripciones, son verdaderas o falsas respectivamente, si lo que precisa ser (hecho) es o no un medio para obtener el fin o resultado pretendido. 
Desde el punto de vista de los sujetos normativos, el fin pretendido que consideraremos es la satisfacción (obediencia) al ordenamiento. Dado que el sujeto precisa obedecer las normas, si el antecedente $a$ de una obligación condicional $\operatorname{Od}(a \rightarrow c)$ es el caso, entonces el sujeto normativo extrae de ese deber deóntico condicional el deber técnico de hacer el consecuente, i. e. $O_{t} c$. Los deberes técnicos extraídos de los deberes deónticos guardan similitud con las propuestas lógicas de separar las obligaciones ideales (deberes deónticos) de las obligaciones actuales (los que si tiene que hacer frente a los factos para que se cumplan las reglas).

Regresando a la paradoja, esto significa que si 4 fuese el caso, entonces tenemos la siguiente regla técnica:

\section{5. $O_{t} q$.}

En lenguaje ordinario, si fuese el caso que «John embaraza a Suzy Mae», entonces: (v) «John tiene que casarse con Suzy Mae» [si quiere satisfacer las normas i)-iv)].

Con este recurso, VON WRIGHT entiende que disuelve la paradoja. Sin embargo, se podría considerar una ulterior objeción, opuesta por HANSEN (HANSEN, 1999), argumentando que de 1, 2 y la separación deóntica, se deriva:

6. $O_{d} \sim q$.

Es cierto que la conjunción de $q \mathrm{y} \sim q$, «casarse y no casarse con Suzy Mae» expresa una contradicción proposicional, pero 5 y 6 todavía no expresan una contradicción normativa ya que los operadores modales son de diferente tipo (uno es deóntico y el otro es técnico). En el abordaje de VON WRIGHT, nada impide que de un deber deóntico «se extraiga» un deber técnico con el mismo contenido. Dado que $O d \sim q$ es el mismo que (es equivalente a) $O d($ taut $\rightarrow \sim q$ ), cuando taut, podemos extraer la siguiente necesidad práctica:

7. $\mathrm{O} \sim \sim$.

Tenemos, por tanto, dos necesidades prácticas inconsistentes y eso, ciertamente, entra en conflicto con nuestras intuiciones, pues no esperamos derivar de i)-iv) que John tiene que casarse y no casarse con Suzy Mae.

Pero, si tomáramos a la realizabilidad (doability) como patrón de racionalidad, es posible, usando un argumento del propio Jorg HANSEN, bloquear el último argumento y evitar el problema. Primero, porque si 4 fuese el caso, entonces la norma 1 es espuria ya que sería irracional exigir a John que regrese en el tiempo y evita el embarazo de Suzy Mae (HANSEN, 1999). Por tanto, bajo esa condición, solo la norma 2 valdría y de ella aisladamente no sería posible derivar 6 . En segundo lugar, porque si 4 fuese el caso, entonces el antecedente de la obligación condicional 2 no sería el caso y, por ello, no surge la necesidad práctica de cumplir lo que tal norma exige, i. e. no casarse con Suzy Mae.

La solución de HANSEN parece estar centrada en el ejemplo específico de la paradoja. PRAKKEN y SERGOT sugieren una formulación de la paradoja de CHISHOLM desprovista de nociones temporales (de modo que la separación entre el tiempo de lo antecedente y del consecuente sea inútil) o de acción (de modo que no funciona la propuesta de limitarse el alcance del operador deóntico a acciones, tomando el antecedente del condicional como una proposición, CASTAÑEDA, 1981). 
i) Prohibido los perros.

ii) Si no hay perros, no debe haber cartel de advertencia.

iii) Si hay perros, debe haber cartel de advertencia.

iv) Hay perros.

Usando LIN, derivamos el deber deóntico «no debe haber cartel de advertencia» (que sería el deber ideal), y también el deber técnico «tiene que haber cartel de advertencia» (que sería la exigencia actual). Mas por la técnica de extracción se obtiene también «que no debe haber cartel de advertencia» y no parece en este ejemplo que la regla ii) sea espuria. Aquí, el problema parece ser que VON WRIGHT admite libremente que los deberes ideales traduzcan exigencias actuales, independientemente de los hechos.

\subsection{Condiciones de adecuación}

En un detallado análisis de las diferentes propuestas de solución para la paradoja de CHISHOlm, CARMO y JONES (CARMO y JONES, 2002) señalan ocho requisitos de adecuación para la formalización de las obligaciones contrarias al deber:

i) Consistencia.

ii) Independencia lógica de las premisas.

iii) Siguiendo a PRAKKEn y SERGOT, aplicabilidad a ejemplos que sean independientes de las diferenciaciones temporales o de la naturaliza de la acción entre antecedente y consecuente.

iv) Estructura similar de formalización para las obligaciones condicionales normales y aquellas contrarias al deber.

v) Aptitud para derivar obligaciones ideales (separación deóntica).

vi) Aptitud para derivar obligaciones actuales (separación fáctica).

vii) Aptitud para representar el hecho de que se ha producido una violación de una obligación.

viii) Aptitud para evitar la «rareza pragmática» (pragmatic oddity), apuntada por PRAKKEN y SERGOT, 1994.

Algunos de estos requisitos merecen comentarios adicionales.

El requisito iv) se opone a la solución propuesta por PRAKKEN y SERGOT (PRAKKEN y SERGOT), en el que usan diferentes formulaciones para las obligaciones en contra del deber. CARMO y Jones (CARMO y JONES, 2002) apuntan a las dificultades que tiene trabajar con esa formalización, en la medida en que cada introducción de nueva norma en una base de datos cuyo antecedente sea la negación del contenido de una de las obligaciones ya existentes exigiría una reformulación de la representación de las normas condicionales de la base.

El requisito vii) se liga al desafío en expresar situaciones de violación de deberes al mismo tiempo que se admite la separación fáctica y deóntica, sin generar a partir del ejemplo un conflicto de obligaciones. El camino propuesto hasta aquí es separar obligaciones actuales de obligaciones ideales, lo que acaba por generar una combinación de modalidades. 
La referencia respecto al requisito viii) es la crítica de PRAKKEN y SERGOT a la solución a la paradoja de CHISHOLM propuesta por JONES y PÖRN (JONES y PÖRN, 1985). JONES y PÖRN separan mundos ideales de mundos actuales que estarían potencialmente en mundos sub-ideales. En su formalización, «Debe haber cartel de advertencia [de la existencia de perros] se deriva de la condición de contrariedad al deber en todas las versiones ideales del mundo actual (de 2 y 4 ) y también que idealmente «No debe haber perros», lo que a pesar de no ser contradictorio, sería más bien extraño. Normalmente, en varios casos una diferenciación temporal de las obligaciones ideales podría dar cuenta del caso, pero el ejemplo presenta una regulación en la que esa diferenciación no procede.

La solución de VON WRIGHT, complementada por el argumento de HANSEN, satisface los requisitos i), ii), iv), v) y vi). Los requisitos v) y vi) pueden admitirse como superados, con el recurso a la derivación de deberes técnicos, que señalan cuáles son las necesidades prácticas actuales, como opuestas a las inferencias de deberes ideales.

Sin embargo, el argumento de HANSEN, al usar la noción de realizabilidad e identificar la primera norma como espuria para impedir la derivación que genera redundancia, traza una distinción temporal, aunque no sea formalmente expresada. Por ello, el argumento no satisface el requisito iii). Tampoco el requisito vii) es bien trabajado ya que aun cuando se obtenga la necesidad práctica adecuada a partir de la condición actual (Suzy Mae está embarazada/hay un perro), subsiste la necesidad práctica de no embarazar a Suzy Mae o de que no hayan perros, con lo que las necesidades prácticas no tratan solamente de las condiciones actuales sino también de las condiciones ideales. Por el contrario, también es posible derivar tanto la necesidad práctica de no embarazar Suzy Mae, a partir de (1), como la necesidad práctica de casarse con ella (de 4 y 3') con la extracción de la necesidad práctica correspondiente a 3», lo que genera la «extrañeza pragmática» y viola viii) desde la perspectiva de las reglas técnicas. Usando el ejemplo de PRAKKEN y SERGOT, tendríamos aquí tanto la necesidad práctica de que no haya perros, como la necesidad práctica de colocar un aviso sobre su existencia.

\subsection{El problema de la inconsistencia racional}

Hay todavía otra objeción más seria y general al tratamiento dado por VON WRIGHT a la lógica deóntica, que acaba por afectar su solución a la paradoja de CHISHOLM.

La obligación condicional 3', Od $(p \rightarrow q)$, es normativamente implicada por 1 $(O d \sim p)$. Pero si eso es verdad, también es verdad, usando las mismas observaciones de PRIOR, que 1 implica normativamente:

3". $\operatorname{Od}(p \rightarrow \sim q)$.

Quiere decir, se deriva que es obligatorio que John no se case con Suzy Mae en caso de que la embarazase. Es claro que eso no significa que «exista» 3' o 3" y no habría problemas con las aparentes paradojas del compromiso (paradoxes of commitment).

Sin embargo, supóngase un legislador, llamado Rex, que aspira a la racionalidad y que, para ello adopta el patrón de racionalidad propuesto por VON WRIGHT, pretendiendo promulgar solamente normas ejecutables. Conforme a ese patrón no hay 
objeciones en promulgar, junto a la norma 1, las normas 3' y 3" conjuntamente. Ambas normas serían ejecutables, conforme al criterio empleado en la definición de inconsistencia deóntica.

Entonces, supóngase que Rex promulga la norma i) y las normas iii') y iii”) como obligaciones contrarias al deber.

i) Es prohibido para John embarazar a Suzy Mae.

ii') Es obligatorio que si John embaraza a Suzy Mae, entonces se case con ella.

iii') Es obligatorio que si John embaraza a Suzy Mae, entonces no se case con ella.

Usando la formalización propuesta por VON WRIGHT, obtenemos la siguiente regulación emitida por Rex:

1. $O d \sim p$.

3'. $\operatorname{Od}(p \rightarrow q)$.

3". $\operatorname{Od}(p \rightarrow \sim q)$.

Supongamos entonces que John embaraza a Suzy Mae:

4. $p$.

Ahora, de 3' y 3" se «extraen» dos necesidades prácticas en conflicto:

5'. Otq.

5". Ot q.

Aquí aparece el problema. La idea fundamental del nuevo abordaje de vON WRIGHT es que el legislador racional jamás podría colocar a los sujetos normativos en una situación en la que cualquier curso de acción que se produzca sea al mismo tiempo «correcto» y «equivocado», ya que fallaría como guía de la conducta de sus súbditos. Pero eso es exactamente lo que ocurre cuando John tiene que ejecutar las necesidades prácticas 5' y 5". Entonces, la regulación 1, 3', 3" debería indicar una irracionalidad en la voluntad de Rex (al menos si 4 fuese el caso), lo que infelizmente no se produce. Rex continua siendo racional si aceptamos el patrón de irracionalidad tal como ha sido propuesto por VON WRIGHT. Llamaremos a esa nueva dificultad «el problema de la inconsistencia racional».

\section{CONSISTENCIA CONDICIONAL}

El problema de la inconsistencia racional es más general que la paradoja de CHISHOLM, dado que se produce tan solo con 3', 3" y 4, sin necesidad de recurrir a una regulación contraria al deber. Sin embargo, esto es crítico para VON WRIGHT ya que no permite una representación satisfactoria de las obligaciones contrarias al deber y, aparte de eso, muestra que el criterio de racionalidad adoptado falla en su propósito, ya que es posible formular una regulación de la que se extraen necesidades prácticas contradictoria, que, a pesar de ello, llevarían el sello de la racionalidad.

De esa manera, VON WRIGHT enfrenta un dilema: o abandona la estrategia de «extracción» de necesidades prácticas a partir de deberes deónticos (y, por tanto, la paradoja de CHISHOLM permanece sin resolución satisfactoria en su lógica de implicación normativa); o abandona el patrón de ejecutabilidad tal como fue definido. 
La raíz del problema está en el conflicto entre la propuesta de formalización de obligaciones condicionales (con el uso de la implicación material en el alcance del operador deóntico) y la definición de consistencia de VON WRIGHT, que no tiene una clausula referida a las condiciones de aplicación de obligaciones o permisiones.

Como consecuencia, la definición de consistencia dice que la conjunción de contenidos normativos de un conjunto de obligaciones no puede representar una contradicción proposicional sin que se comprometa la racionalidad del legislador. Pero, como la obligación condicional se formaliza por medio de la implicación material, $O_{d}(a \rightarrow b)$, el contenido de tal obligación es dado por el estado de cosas $(a \rightarrow b)$ y no se hace explícito que $a$ sea una condición para la obligación $O_{d} b$. Como en la lógica proposicional clásica, $a \rightarrow b$ no contradice al estado $a \rightarrow \sim b$, entonces el conjunto $N=\left\{O_{d}(a \rightarrow b)\right.$, $\left.O_{d}(a \rightarrow \sim b)\right\}$ es resultado de la legislación racional, en virtud del criterio de consistencia de VON WRIGHT.

Hay que notar que la negación aplicada al contenido de la obligación (permisión) condicional no distingue la «condición de aplicación» del «alcance» de la obligación. Si el alcance de la obligación es una implicación material y se pretende decir que solo el consecuente es obligatorio hay un precio que pagar con relación a la extensionalidad del lenguaje, i.e. con relación a la posibilidad de la sustitución de equivalentes.

Por ejemplo, sabemos que $a \rightarrow \sim b$ es equivalente en la lógica clásica a $\sim(a \wedge b)$. Si en la primera formulación, el alcance de la obligación fuese $\sim b$, dada su estructura condicional eso no sería el caso en la segunda que, a pesar de tener el mismo significado, no tiene estructura condicional. Por ello, si alteramos la definición de consistencia o la noción de norma-negación de forma que se aplicase solamente al consecuente como alcance de la obligación deóntica, entonces, $N=\left\{O_{d}(a \rightarrow b), O_{d}(a \rightarrow \sim b)\right\}$ seria inconsistente, pero $N=\left\{O_{d}(a \rightarrow b), O_{d}(\sim(a \wedge b))\right\}$ no lo sería. Eso significa que la formulación lingüística de la norma pasaría a tener relevancia para la noción de conflicto normativo y, consecuentemente, para la noción de implicación entre normas (dado que esta última es definida a partir de aquella).

En la formulación original de su sistema de lógica deóntica diádica, VON WRIGHT admitió una forma de interdefinibilidad entre permisión y obligación condicional, introduciendo el operador $\mathrm{P}$ de permisión por medio de la definición $P(a / b)=\sim O(\sim a / b)$, es decir, «a es permitido si $b$ fuese el caso» significa lo mismo que « $a$ no es prohibido si $b$ fuese el caso».

La interdefinibilidad entre permisión y prohibición se liga al postulado de completitud, dado que es necesaria para derivar $O a / b \vee O \sim a / b \vee(P a / b \vee P \sim a / b)$, esto es, para una condición $b$ cualquiera, cualquier estado $a$ es obligatorio, prohibido o facultativo. Postular que no hay contradicción entre obligaciones y prohibiciones $\sim(O a / b \vee O \sim a / b)$ merece más atención. Si lo aceptasemos, junto con el postulado intuitivo del refuerzo del antecedente $(O a / b \rightarrow O a / b \wedge c)$, esto es, que « $\langle b »$ es una condición suficiente para la obligación de «a», aparece una dificultad para la representación de las obligaciones contrarias al deber respecto a condiciones independientes. Ello ocurre porque $(\mathrm{Oa} /$ $b \wedge O \sim a / c)$ se deriva $(O a / b \wedge c) \wedge(O \sim a / b \wedge c)$.

Esta constatación fue una divisoria entre dos tradiciones en lógica deóntica díadica: la tradición de VON WRIGHT $(1964 ; 1965)$, que niega el postulado de no contra- 
dicción para las obligaciones condicionales y la tradición de HANSSON (1971), que abandona la regla del fortalecimiento del antecedente.

Por otro lado, el postulado de no contradicción para una misma condición, tampoco consiente la reducción del condicional deóntico a la implicación material.

Aún más, un problema ligado al uso de la implicación material, en el último abordaje de VON WRIGHT, aparece en el método propuesto para la separación fáctica por medio de la extracción de deberes técnicos, que son necesidades prácticas para el cumplimiento de las prescripciones condicionales.

Jorg HANSEN argumenta que, desde el punto de vista de los sujetos normativos, una obligación de la $O_{d}(a \rightarrow b)$ puede ser obedecida evitando que $a$ sea el caso. En otras palabras, la obligación genera no solo una necesidad práctica de hacer $b$ si $a$ fuese el caso, $i$. e. se extrae no solo $a \rightarrow O_{t} b$, sino también una necesidad práctica de que $\sim a$ sea el caso, i. e. se extrae también $O_{t} \sim a$. Ahora bien, per se, esto no llega a ser problemático, pero abre algunas puertas para una serie de ejemplos contra-intuitivos. Así, en el ejemplo del kindergarten de HANSEN, tenemos que de la obligación del director de una escuela pública de garantizar una plaza para cada niño nacido en una cierta comunidad, ipuede extraerse la necesidad práctica de distribuir anticonceptivos!

VON WRIGHT, ciertamente, permite esa interpretación cuando concede que de $O d a$ se puede extraer taut $\rightarrow O_{t} a \mathrm{y}$, consecuentemente, $O_{t} a$. Por tanto, también es posible extraer de $O_{d}(a \rightarrow b)$, la necesidad práctica $O_{t}(a \rightarrow b)$. Entonces, hacer que $\sim a$ sea el caso es un medio para satisfacer esa necesidad práctica. Pero, al mismo tiempo, vON WRIGHT reconoce que al similar al ejemplo del kindergarten no sería una «reacción apropiada a la prescripción» (HANSEN, 2001).

Es posible reconstruir el método de extracción de necesidades prácticas con cláusulas recursivas que permitan separar únicamente el consecuente de la implicación material (MARANHãO, 2010). Sin embargo, al hacerlo de ese modo, se desnaturaliza una vez más la implicación material, como tal, para los deberes técnicos.

Por ello, para lidiar con el problema de la inconsistencia racional y el del Kindergarten de HANSEN, exploraremos una versión diádica de la lógica de la implicación normativa.

\section{LÓGICA DE IMPLICACIÓN NORMATIVA DIÁDICA}

Para obtener el lenguaje de $\operatorname{LIN}_{d y}$ basta sustituir la cláusula de los operadores deónticos en la definición de FN por

i) Si $a$ y $b$ son contenidos normativos (i. e. $a, b \in F C$ ) entonces, $O a / b$ y $P a / b$ son formulaciones normativas (i. e. $\mathrm{O} a / b, \mathrm{~Pa} / \mathrm{b} \in \mathrm{F}_{N}$ ).

Así como ocurre en LIN, no son bien formadas sentencias deónticas con conectivos booleanos fuera del alcance de los operadores modales. Se definen los operadores monádicos de modo usual:

$$
\begin{aligned}
& \mathrm{O} a=d f \text { Oa/taut } \\
& \mathrm{Pa}=d f \mathrm{~Pa} / \text { taut }
\end{aligned}
$$


Juliano Maranhão

Para superar el problema de la inconsistencia racional, además de la interdefinibilidad entre permisión y obligación diádica, la lógica de la implicación normativa diádica tendría que validar un postulado análogo al esquema $\mathrm{D}$ :

(D) $O(a / b) \Rightarrow_{d y} P(a / b)$

En el abordaje de VON WRIGHT, el esquema no significaría que existiese un permiso condicional con tal efecto en el ordenamiento, sino solo que, si el legislador exige la realización de $a$ cuando $b$ fuese el caso, sería irracional exigir $\sim a$ para esa misma condición, lo que parece intuitivo.

Para validar $\mathrm{D}$, tenemos que proceder a la siguiente reconstrucción de las definiciones claves de VON WRIGHT. Primero, para un conjunto $\mathrm{N}$ de normas, definimos su «cuerpo» de condiciones y su «cabeza» de consecuencias.

$N b=\{x: O(b / x)$ o $P(b / x) \in N\}$

$N b=\{y: O y / b$ o $O y / b \in N\}$

En seguida, se define una versión diádica de inconsistencia de un conjunto de normas, ligado a una determinada condición.

Definición de inconsistencia diádica. Sea $N \subseteq F_{N}$ y $b \in F C$. Entonces, $N$ es dy-inconsistente con respecto a $b\left(i n c_{d y}(N / b)\right)$ si, y solamente si, existe $M=\left\{O a_{1} / b_{1}, \ldots, O a_{n} /\right.$ $\left.b_{n}\right\} \subseteq N$ o $M=\left\{O a_{1} / b_{1}, \ldots, O a_{n} / b_{n}, P x / y\right\} \subseteq N$ tal que: i) $b \Rightarrow \wedge\left(M_{b}\right)^{3}$ y ii) $M b \Rightarrow \perp$. En caso contrario $N$ es dy-consistente para $b$.

Hay que destacar que la condición de la norma derivada a ser testeada determina las reglas que son «activadas». Las reglas «activadas» son aquellas que tienen su antecedente derivado por i). Solamente las reglas activadas tienen su consecuente testado como la negación hipotética de la norma derivada, conforme con la cláusula ii). Con estas cláusulas, la definición se aproxima al mecanismo de inferencia usado por MAKINSON y VAN DER TORRE para las lógicas input/output (MAKINSON y VAN DER TORRE, 2000), pero ya se incluye aquello que sería el «generador» de consecuencias, tanto obligaciones como permisiones. Recordemos que aquí, el test no se interpreta como generador de consecuencias, ya que las reglas derivadas no tienen estatus ontológico propio, sino que solo indica que sería irracional regular la conducta en sentido opuesto.

El problema de la inconsistencia racional es removido con esta definición. Basta verificar que $\left.\operatorname{inc}_{d y}(\{O(\sim a / b), O(a / b)\} / b)\right)$. Es posible incrementar la exigencia de que la condición determinante para el test de consistencia no sea una contradicción, con el objetivo de evitar que una regulación como $\{O(\sim a / b), O(a / \sim b)\}$, que atribuye obligaciones contrarias a situaciones mutuamente excluyentes, sea considerada una forma de legislación irracional para $\mathrm{b} \wedge \sim \mathrm{b}$. O sea, evitar que valga inc $\mathrm{d}_{d y}(\mathrm{~N} / \perp)$ sse $\mathrm{N} b \Rightarrow \perp$. Como el objetivo del test de consistencia sería determinar cuáles son obligaciones legislables

${ }^{3} \wedge\left(M_{b}\right)$ es la conjunción de todos los elementos de $M_{b}$. 
para determinadas condiciones hipotéticas, una condición hipotética contradictoria sería simplemente excluida como una posibilidad de derivación: no cabe validar la racionalidad del legislador con hipótesis irracionales

Introducimos, entonces, la función de negación en el lenguaje diádico:

$$
\begin{aligned}
& { }_{d y} n e g((O(b / a))=d f P(\sim b / a) \\
& { }_{d y} n \operatorname{eg}((P(b / a))=d f O(\sim b / a)
\end{aligned}
$$

Ahora definimos la implicación diádica, haciendo que la condición de la norma derivada determine el tipo de inconsistencia testeada para la base de normas consideradas, esto es:

Definición de implicación deóntica diádica $\left(\Rightarrow_{d y}\right)$ : Considérese $N \subseteq F N$ y $\Delta(a / b) \in F_{N}$, donde $\Delta \in\{O, P\}$. Entonces $N \Rightarrow_{d y} \Delta(a / b)$ si, y solamente si, inc $c_{d y}\left(N \cup\left\{_{d y} n e g(\Delta(a / b)\} / b\right)\right.$.

Como se puede verificar fácilmente, $\operatorname{LIN}_{d y}$ confirma el esquema $\mathrm{D}$, junto a los siguientes esquemas estructurantes de la lógica deóntica diádica:

(CO) $O(a \rightarrow b / c), O(a / c) \Rightarrow_{d y} O(b / c)$ (principio de consecuencia de obligaciones)

(AO) $O(a / c), O(b / c) \Rightarrow_{d y} O(a \wedge b / c)$ (principio de agregación de obligaciones)

Esos dos principios muestran que, fijada una condición, la «base» del cuerpo de reglas operará de forma similar a la versión monádica de LIN. Dada una cierta condición, las consecuencias lógicas de las obligaciones son obligatorias, en el sentido de que permitir su omisión será irracional. También vale en $\operatorname{LIN}_{d y}$, el principio del refuerzo del antecedente, lo que junto a $\mathrm{D}$, trae nuevamente el dilema que separo las dos tradiciones en la lógica deóntica diádica.

(SA) $O(a / b) \Rightarrow_{d y} O(a / b \wedge c)$

Las reglas que están más abajo, válidas en $\operatorname{LIN}_{d y}$, muestran que la forma lógica de las condiciones de las normas y de su contenido (alcance) no es irrelevante

Si $\varnothing \Rightarrow a \leftrightarrow b$, entonces $O(c / a) \Rightarrow_{d y} O(c / b)$ y $O(c / b) \Rightarrow_{d y} O(c / a)$

Si $\varnothing \Rightarrow a \leftrightarrow b$, entonces $O(a / c) \Rightarrow_{d y} O(b / c)$ y $O(b / c) \Rightarrow_{d y} O(a / c)$

\section{LA PARADOJA DE CHISHOLM Y LA LÓGICA DE LA IMPLICACIÓN NORMATIVA DIÁDICA IDEALIZADA}

A primera vista, LIN $_{d y}$ parece inadecuada frente al ejemplo de CHISHOLM, por el simple hecho de que no admite separación fáctica, ni deóntica. En efecto, en $\operatorname{LIN}_{d y}$ no valen:

(DD) $\mathrm{O} a, \mathrm{O}(b / a) \Rightarrow_{d y} \mathrm{Ob}$

(FD) $a, O(b / a) \Rightarrow_{d y} \mathrm{O} b$

Hay que notar que FD ni tan siquiera es una fórmula bien formada, ya que $\operatorname{LIN}_{d y}$ opera solamente con formulaciones normativas, y que DD no se sigue ya que una 
condición determinante del test de consistencia es taut, de modo que la premisa condicional no es activada.

Sin embargo, recordemos que al igual que LIN, $\mathrm{LIN}_{d y}$ no contempla supuestos ontológicos acerca de las normas derivadas. Estas solo representan cuestiones acerca de que sería racional exigir o permitir a partir de ciertas premisas normativas. El objetivo primordial de VON WRIGHT con LIN era proporcionar lo que WITTGENSTEIN llamaba presentación panorámica del uso ordinario del lenguaje, es decir, presentación de conexiones desapercibidas en el lenguaje ordinario, que pueden evidenciar naturalmente que el problema filosófico no se presenta. En relación con CHISHOLM, la propuesta de VON WRIGHT consistía en diferenciar el contexto ideal del contexto actual, mediante la distinción entre deberes deónticos y técnicos. Los deberes deónticos parten de la perspectiva del legislador que, se asume idealmente, pretende ver obedecidas a sus ordenes y aprovechados sus permisos y, como mínimo de racionalidad, desea ofrecer un conjunto de obligaciones y permisiones realizables. Los deberes técnicos son considerados desde la óptica de los sujetos normativos, que se preguntan qué, de hecho, tienen que hacer (necesidades prácticas) para obedecer las órdenes y aprovechar los permisos. Hay que recordar asimismo que LIN no permite la separación fáctica, lo que ocurre solamente en el contexto actual de los deberes técnicos y de la perspectiva de los sujetos normativos.

Por ello, en un contexto deónticamente ideal, se asume que se cumple con todas las obligaciones que se tienen oportunidad de cumplir sin que se impida un aprovechamiento de cada uno de los permisos. Sobre la base de este presupuesto se erige el test de consistencia, lo que daría una variación $\mathrm{LIN}_{d y}$, que podemos llamar «lógica de implicación normativa diádica ideal», LIN $_{i d y}$.

En $\operatorname{LIN}_{i d v}$, la idea básica es sumar a la condición $b \in F c$ que determina el test de consistencia, la aserción de que el contenido de las obligaciones y de cada una de las permisiones contenidas en un conjunto $N$ es satisfecho en relación a las oportunidades creadas por la condición determinante. Para la verificación de las reglas son «activadas» por la condición determinante y el cumplimiento de las obligaciones vigentes obviamente no se considera a la norma derivada hipotética cuya racionalidad se pretende poner a prueba. Esta salvaguarda tiene el inconveniente de vincular el test de consistencia a la norma derivada cuya racionalidad se indaga. La implicación es definida a partir de un conjunto de normas (vigentes), una norma (implicada) y determinada condición (respecto de la norma implicada).

Definición de implicación deóntica diádica idealizada $\left(\Rightarrow_{i d y}\right)$.

Considérese $N \subseteq F_{N}, \Delta(a / b) \in F N$, donde $\Delta \in\{O, P\}$. Entonces:

a) $\mathrm{N}$ es idy-inconsistente con respecto a $b\left(i n c_{i d y}(\mathrm{~N} / b)\right)$ si y solamente si, existe $M=\left\{O a_{1} / b_{1}, \ldots, O a_{n} / b_{n}\right\} \subseteq N$ o $M=\left\{O a_{1} / b_{1}, \ldots, O a_{n} / b_{n}, P x / y\right\} \subseteq N$ tal que i) (M/ $\left.{ }_{d y} n e g \Delta(a / b)\right) b \cup b \Rightarrow \vee(M b)$ y ii) $M b \Rightarrow \perp$.

En caso contrario, $\mathrm{N}$ es idy-consistente para $b$.

b) $\quad N \Rightarrow{ }_{i d} \mathrm{y} \Delta(a / b)$ si y solamente si $i n c_{i d y}\left(N \cup\left\{{ }_{d y} n e g(\Delta(a / b)\} / b\right)\right.$. 
Es fácil verificar que verificar que en $L I N_{i d y}$ vale DD.

Se puede decir que en un contexto ideal, en $L I N i_{d y}$, se asume que las obligaciones son cumplidas. Este presupuesto, sin embargo, puede ser derrotado frente a los contextos actuales, que es justamente lo que ocurre con la paradoja de CHISHOLM, que se retoma abajo en la versión de PRAKKEN y SERGOT:

\begin{tabular}{|ll|l|}
\hline 1. & Prohibido los perros. & $O \sim p$ \\
\hline 2. & Si no hay perros, no debe haber un cartel de advertencia. & $O(\sim a / \sim p)$ \\
\hline 3. & Si hay perros debe haber un cartel de advertencia. & $O(a / p)$ \\
\hline
\end{tabular}

En $\operatorname{LIN}_{i d y}$, se obtiene:

$$
1,2,3 \Rightarrow_{i d y} \mathrm{O} \sim \mathrm{a}
$$

El significado de esta derivación es que sería irracional permitir el cartel de advertencia sobre perros si es que están prohibidos los perros, cuando se asume que el deber fue cumplido. Por otro lado, en $\operatorname{LIN}_{i d y}$, no es irracional (resultado de la legislación inconsistente), el conjunto de las tres reglas 1,2,3 (en el caso en que se excluyan las condiciones contradictorias para el test de consistencia, como fue sugerido anteriormente).

Ese es el contexto ideal de preguntas de acuerdo con la perspectiva de la racionalidad del legislador. Hay que notar que en la lógica de la implicación normativa idealizada, no entran en juego las premisas fácticas.

Sin embargo, en el cuadro de necesidades práctica para el sujeto normativo para cumplir con el ordenamiento, la pregunta es acerca de cuáles reglas compatibles con el contexto actual pueden ser cumplidas, lo que significa la «extracción» de deberes técnicos (actuales) debe partir de una restricción al conjunto original de obligaciones/ permisiones. La restricción obvia consiste en no considerar reglas que deriven obligaciones inconsistentes con la premisa fáctica (en concreto, esas no pueden ser cumplidas). De ese modo, se define la función de extracción de deberes técnicos «Ot» para un conjunto de normas, con ayuda de un operador de contracción.

Definición de conjuntos normativos saturados. Dado un conjunto de normas $N \subseteq F N$ y una premisa fáctica $b \in F C$, la familia de conjuntos normativos b-saturados de $N$ es dado por $\operatorname{sat}(N, b)=\left\{N^{\prime} \subseteq N\right.$; no ocurre $N^{\prime} \Rightarrow i_{d v} O \sim b$; y $N^{\prime}$ es el mayor conjunto que satisface esas condiciones\}.

Nótese que todo $\mathrm{N}^{\prime} \in \operatorname{sat}(\mathrm{N}, \mathrm{b})$ es normativamente consistente.

Definición de la función de extracción de deberes técnicos. Dado un conjunto de normas $N \subseteq F N$ y una premisa fáctica $b \in F C$. Entonces, la función de extracción $O t$ : $\mathrm{P}\left(F_{N}\right) \times F_{C} \rightarrow P\left(F_{C}\right)$ es tal que $O t(N, b)=\left\{x: \mathrm{N}^{\prime} \Rightarrow i_{d y} O x / y\right.$, tal que $b \Rightarrow y$ e $\left.\mathrm{N}^{\prime} \in \operatorname{sat}(\mathrm{N}, \mathrm{b})\right\}$.

O sea, la función de extracción selecciona un conjunto dentro de una familia de subconjuntos normativos saturados (que tienen un contenido obligacional consistente) en relación al contexto fáctico que se presenta. De ese conjunto normativo saturado, solo el contenido de obligaciones aplicables al contexto fáctico necesitan ser cumplidas, i. e. constituyen deberes técnicos. 
Retomando el ejemplo de Prakken y SERGot, se obtiene que $O t(N, p)=\{a\}$, o sea, la necesidad práctica para el caso de que haya perros es que haya cartel de advertencia.

Por otro lado, $O t(N, \sim p)=\{\sim p, \sim a\}$, lo que muestra el hecho de que para los casos en los que no hay inconsistencia entre los contenidos normativos del ordenamiento $N$, el conjunto de deberes técnicos corresponde al conjunto de deberes deónticos monádicos derivados de $N$ en $L I N i_{d y}$, es decir, si $\cap \operatorname{sat}(N, b)=\{N\}$, entonces $O t=\left\{x: N \Rightarrow i_{d y}\right.$ $O x\}$. En la hipótesis de conflicto entre el conjunto de normas $N$ y la premisa fáctica $y$, se obtiene $\cap \operatorname{sat}(N, y) \subset N$.

\section{CONCLUSIÓN}

El desarrollo de $\operatorname{LIN}_{i d y}$, junto a la función de extracción de deberes técnicos $O t$, tuvo por objetivo evitar el problema de la inconsistencia racional y el del Kindergarten. Acerca de la inconsistencia racional, el problema estaba en la caracterización de las exigencias en conflicto bajo una misma condición como algo que podría ser el resultado de una legislación racional cuando, desde el punto de vista de los sujetos normativos, se generarían dos necesidades prácticas que no pueden ser realizadas bajo esa condición. Con relación al Kindergarten, el problema estaba en la posibilidad de que los sujetos normativos atendiesen la exigencia práctica de una norma condicional impidiendo la ocurrencia del antecedente. Al mismo tiempo, el objetivo era mantener la interpretación del sistema lógico siguiendo una intuición no comprometida con el status ontológico de las normas derivadas, que fuese capaz de distinguir deberes deónticos derivados como hipótesis de racionalidad del legislador de los deberes técnicos en tanto que necesidades prácticas extraídas de los ordenamientos. Por otro lado, la solución de VON WRIGHT para la paradoja de CHISHOLM, al igual que la reconstrucción de HANSEN, parecen asumir una diferencia temporal.

Como hemos visto anteriormente, $\operatorname{LIN}_{i d y}$, junto a la función de extracción de deberes técnicos $O t$, supera el problema de la inconsistencia racional y del Kindergarten. En relación a los criterios de adecuación de CARMO y JONES (2002), hay que señalar lo siguiente:

i) Las premisas son consistentes, en el sentido de que el conjunto normativo de Chisholm no representa el resultado de una legislación racional, siempre que la condición determinante del test de consistencia no sea contradictoria.

ii) Las premisas son lógicamente independientes entre sí, teniendo en cuenta que solamente se derivan de conjuntos normativos (de modo que la premisa fáctica no contribuye en cualquier derivación normativa).

iii) La solución se aplica a ejemplos independientes de diferenciaciones temporales o de la naturaleza de la acción que enlaza antecedente y consecuente.

iv) Las obligaciones deónticas en condiciones normales y aquellas que surgen en condiciones contrarias a deber reciben la misma formalización en $L I N_{i d \gamma}$.

v) En $L I N_{i d y}$ se pueden derivar obligaciones ideales (separación deóntica).

vi) La función $O t$ tal como definida en $\operatorname{LIN}_{i d y}$ admite derivar obligaciones actuales (separación fáctica). 
vii) El hecho de que se ha producido una violación de una obligación está representado por la hipótesis en que es el caso que $O t(N, y) \neq\left\{x: N \Rightarrow_{i d y} O x\right\}$, que es un caso en el que $y$ constituye una violación del conjunto de normas y, por tanto, $\cap \operatorname{sat}(N, y) \subset N$.

viii) La «rareza pragmática» no emerge en $\operatorname{LIN}_{i d \gamma}$ ya que solo se deriva una necesidad práctica de tener en cuenta al contenido de la obligación contraria al deber y no un deber deóntico, que pudiese colocarse junto al deber deóntico violado. O sea, de acuerdo con el ejemplo de PRAKKEN y SEGOT, no se sigue en $L I N_{i d y}$ que hay un deber deóntico de que no haya perros junto al deber de colocar un cartel de advertencia. Frente al hecho de que hay un perro se deriva solo el deber deóntico de que no haya perros (i.e., el legislador no podría racionalmente permitir perros) y la necesidad práctica de colocar un cartel que advierta de que hay perros, si los hubiese.

\section{BIBLIOGRAFÍA}

AlChOURRÓN, C., 1993: «Philosophical foundations of Deontic Logic and the Logic of Defeasible Conditionals», en J.-J. Ch. MeYER y J. R. WIERINGA (eds.), Deontic Logic in Computer Science: Normative System Specification, John Wiley \& Sons, 43-84.

ÅQvIST, L., 1987: «An Introduction to Deontic Logic and the Theory of Normative Systems», en Indices, Monographs in Philosophical Logic and Formal Linguistics, Naples: Bibliopolis.

CARMO, J., y JoneS, A. J. I., 2002: «Deontic Logic and contrary-to-duties», en D. GABBAY y F. GUENTHNER (eds.), Handbook of Philosophical Logic, vol. 8, 265-343.

CastañEDA, H.-N., 1981: «The Paradoxes of Deontic Logic: The Simplest Solution to All of Them in One Fell Swoop», en R. Hilpinen (ed.), New Studies in Deontic Logic, Dordrecht, Reidel, 37-86.

Chisholm, R. M. , 1963: «Contrary to Duty Imperatives and Deontic logic», Analysis, 24, 33-36.

HAnsen, J., 1999: «Are there Paradoxes of Commitment in Professor von Wright's Logic of Norms?», en G. MegGLE (ed.), Action, Norms, Values: discussions with Georg Henrik von Wright, Walter de Guyter.

Hansson, B., 1971: «An analysis of some deontic logics», en R. Hilpinen (ed.), Deontic Logic: introductory and systematic readings, Dordrecht, D. Reidel, 121-147.

Hilpinen, R., y FollesDal, D., 1971: «Deontic Logic: an introduction», en R. Hilpinen (ed.), Deontic Logic: Introductory and Systematic Readings, D. Reidel Publishing Company.

JørGENSEN, J., 1937-1938: «Imperatives and Logic», Erkenntnis, 7: 288-296.

JONES, A. I., y PORN, I., 1985: «Ideality, sub-ideality and deontic logic», Synthese, 65, 275-290.

LOWER, B., y BELZER, M., 1983: «Dyadic deontic detachment», Synthese, 54, 295-318.

MAKINSON, D., 1999: «On a fundamental problem of deontic logic», en P. MCNAMARA y PraKKEN (eds.), Norms, Logics and Information Systems, Amsterdam, IOS.

MAKINSON, D., y VAN DER TORRE, L., 2000: «Input/Output Logics», Journal of Philosophical Logic, 29, 383-408.

MARANHÃO, J., 2002: «Von Wright's sceptic turn on the logic of norms and the problem of gaps in normative systems», en Archiv fur Rechts- und Sozialphilosophie-ARSP, vol. 88, Heft. 02, 217-229.

- 2009: «Von Wright's Therapy to Jorgensen's Syndrome», en Law and Philosophy, 28: 163 201, Springer.

— 2010: «Condicionalidade e a lógica de implicação normativa de von Wright», Revista Brasileira de Filosofia. 
Prakken, H., y Sergot, M. J., 1996: «Contrary-to-duty obligations and defeasible deontic reasoning», Studia Logica, 57, 91-115.

- 1997: «Dyadic deontic logic and contrary-to-duty obligations», en D. NuTE (ed.), Defeasible Deontic Logic, Synthese Library, 223-262.

Ross, A., 1941: «Imperatives and Logic», Theoria, 7, 53-71.

VON Wright, G. H., 1951: «Deontic Logic», Mind, 60:1-15.

- 1964: «A New System of Deontic Logic», Danish Yearbook of Philosophy, vol. 1, 173-182.

- 1965: «A Correction to a New System of Deontic Logic», Danish Yearbook of Philosophy, vol. 2, 103-107.

- 1983: «Norms, Truth and Logic», en Practical Reason. Philosophical Papers, vol. 1, Oxford: Basil Blackwell, 130-209.

- 1985: «Is and Ought», en E. Bulygin (ed.), Man, law and other forms of life, Reidel, 263-281.

- 1991: «The Foundations of deontic logic- a critical survey», en J. J. MEYER y WiERINGA (eds.), Proceedings of $\triangle E O N ' 91$.

- 1991: «Is there a logic of Norms?», Ratio Juris, 4: 265-283.

- 1996: «Referências são feitas à versão reimpressa», en Six Essays in Philosophical Logic, Acta Philosophica Fennica, vol. 60.

- 1997: «On Norms and Normpropositions», en The Reasonable as Rational?, Festschrift in honour of Aulis Aarnio, ed. Werner Krawietz u.a.. Dunker \& Humboldt. 1997.

- 1999a: «Deontic Logic: as I see it», en P. McNamara y H. Prakken (eds.), Norm Logics and Information Systems ( $\left.\triangle E O N^{\prime} 98\right)$, Amsterdam IOS, 15-25.

— 1999b: «Value, Norm and Action in my Philosophical Writings», en G. MEgGLE (ed.), Action, Norms, Values: discussions with Georg Henrik von Wright, Walter de Guyter. 